\title{
Collective modes across the soliton-droplet crossover in binary Bose mixtures
}

\author{
Alberto Cappellaro, ${ }^{1, *}$ Tommaso Macrì ${ }^{2, \dagger}$ and Luca Salasnich ${ }^{1,3, \dagger}$ \\ ${ }^{1}$ Dipartimento di Fisica e Astronomia “Galileo Galilei,” Università di Padova, Via Marzolo 8, 35131 Padova, Italy \\ ${ }^{2}$ Departamento de Física Teórica e Experimental, Universidade Federal do Rio Grande do Norte, and International Institute of Physics, \\ Natal, 59078-970 Rio Grande do Norte, Brazil \\ ${ }^{3}$ CNR, INO, Via Nello Carrara 1, 50019 Sesto Fiorentino, Italy
}

(Received 24 March 2018; published 29 May 2018)

\begin{abstract}
We study the collective modes of a binary Bose mixture across the soliton to droplet crossover in a quasione-dimensional waveguide with a beyond-mean-field equation of state and a variational Gaussian ansatz for the scalar bosonic field of the corresponding effective action. We observe a sharp difference in the collective modes in the two regimes. Within the soliton regime, modes vary smoothly upon the variation of particle number or interaction strength. On the droplet side, collective modes are inhibited by the emission of particles. This mechanism turns out to be dominant for a wide range of particle numbers and interactions. In a small window of particle number range and for intermediate interactions, we find that monopole frequency is likely to be observed. We focus on the spin-dipole modes for the case of equal intraspecies interactions and equal equilibrium particle numbers in the presence of a weak longitudinal confinement. We find that such modes might be unobservable in the real-time dynamics close to the equilibrium as their frequency is higher than the particle emission spectrum by at least one order of magnitude in the droplet phase. Our results are relevant for experiments with two-component Bose-Einstein condensates for which we provide realistic parameters.
\end{abstract}

DOI: 10.1103/PhysRevA.97.053623

\section{INTRODUCTION}

The exquisite control of parameters in ultracold quantum gases, culminating with the achievement of Bose-Einstein condensation in alkali-metal vapors, boosted a great experimental effort to engineer multicomponent systems [1-3]. From the theoretical standpoint, the spinorial nature of the order parameter and its complex dynamics, as in ${ }^{3} \mathrm{He}-{ }^{4} \mathrm{He}$ mixtures and three-fluid models [4-6], enable an ample variety of interesting physical effects. They span from persistent supercurrent and internal Josephson effect [7-9] and itinerant ferromagnetism in fermionic mixtures $[10,11]$ to more exotic topics such as the research for analogs of Hawking radiation in bosonic mixtures [12-14] and spin-orbit physics [15-18].

More recently, the theoretical proposal of the existence of self-bound quantum droplets in binary bosonic mixtures by Petrov and Astrakharchik [19,20] offered the opportunity for the investigation of nontrivial quantum phases in BoseEinstein condensates (BECs). Dilute quantum droplets were first observed in a single-component dipolar condensate of dysprosium [21-23] even in the absence of external confinement [24], confirming droplet self-stability. Experiments with binary mixtures were recently performed using two internal states of ${ }^{39} \mathrm{~K}$ by Cabrera and co-workers $[25,26]$ and Semeghini et al. [27] both in three dimensions and in quasi-one-dimensional setups. Theoretical work on both dipolar [28-32] and binary BECs [33-35] describes well such experiments in a variety of

\footnotetext{
*cappellaro@pd.infn.it

†macri@ fisica.ufrn.br

†luca.salasnich@unipd.it
}

regimes by the inclusion of leading Gaussian corrections to the mean-field equation of state.

In a recent experiment [26], the formation of dilute selfbound states in a two-component BEC was studied in a tight optical waveguide. Interestingly, above a critical value of the magnetic field a smooth crossover interpolating between droplets and bright soliton states was observed. Below such a critical magnetic field and for small particle numbers a bistable region is detected, corresponding to different minima of the energy functional. Importantly, droplets appear naturally as a competition between mean-field and quantum fluctuation energies with opposite sign. Solitons, on the other hand, are excitations appearing genuinely in low-dimensional systems. If the one-dimensional (1D) interaction strength is repulsive (self-defocusing nonlinearity) one recovers localized dark solitons, while for attractive condensates (self-focusing nonlinearity) one finds bright solitons. Both dark and bright solitons have been observed experimentally with atomic BECs [36].

In this work we study the collective modes of a twocomponent Bose gas in an optical waveguide along the crossover from solitons to droplets. Collective modes are an essential tool in identifying and characterizing the behavior of a quantum system both at zero and at finite temperature. For a single-component BEC with contact interactions in a confining potential they have been studied thoroughly both theoretically and experimentally [37]. In the presence of nonlocal interactions, such as soft-core [38-41] or dipolar potentials $[42,43]$, or internal coupling in multicomponent systems [33,34], collective modes turn out to be crucial in detecting quantum phase transitions from the uniform state to a structured ground-state configuration. Binary systems 
naturally support modes where the two components move in phase, such as monopole and quadrupole oscillations, which are expected to be the lowest-energy excitations. In these simple cases the two components share the same spatial wave function over the oscillation period. More interestingly, there exist cases in which the spinorial nature of the order parameter allows nontrivial collective modes where the internal components move out of phase around the equilibrium configuration. The simplest case is represented by the so-called spin-dipole excitation. For a repulsive two-component Bose gas the spin-dipole oscillation frequency depends crucially on the presence of an external confining potential as well as on the reciprocal interaction strength and was recently characterized in an experiment of Bienaimé et al. [44]. Remarkably, for attractive binary mixtures, spin-dipole oscillations may take place even in the absence of an external potential, the restoring force being proportional to the interspecies interaction. In this paper we characterize such modes, highlighting the combined effect of the standard mean-field attraction and the beyondmean-field corrections to the oscillation frequency. We employ a variational approach based on a Gaussian ansatz for the space modes of the two components, which has been proven to perform well close to the equilibrium configuration. We find striking differences in the collective modes between droplets and solitons. Namely, in the soliton phase we are able to generalize well-known results of quasi-1D BECs. In the droplet regime we find that collective modes are generically inhibited as they have energies higher than the particle emission spectrum for a wide range of available experimental parameters.

In Sec. II we summarize the formalism and the variational results of the ground state of the two-component mixtures with equal masses. In Sec. III we derive the collective modes in the crossover from soliton to droplet for a wide range of particle numbers and interaction strengths for the experimentally relevant case of ${ }^{39} \mathrm{~K}$ within the variational approach. In Sec. IV we focus on the spin-dipole oscillation mode, which is characteristic of a two-component mixture. We specialize to the case of equal intraspecies interaction strength for different particle numbers and compare it with the particle emission spectrum. In Sec. V we highlight the connection of the present study with current experiments on mixtures and give some perspectives for future work.

\section{SOLITON-TO-DROPLET CROSSOVER}

We begin by considering a Bose gas made of atoms in two different hyperfine states in a volume $L^{3}$. Each component can be described by a complex field $\psi_{i}(i=1,2)$, whose dynamics results from the real-time low-energy effective action

$$
S=\int d t d^{3} \mathbf{r}\left[\sum_{j=1,2} \frac{i \hbar}{2}\left(\psi_{j}^{*} \partial_{t} \psi_{j}-\psi_{j} \partial_{t} \psi_{j}^{*}\right)-\mathcal{E}_{\mathrm{tot}}\left(\psi_{1}, \psi_{2}\right)\right] .
$$

The total energy density $\mathcal{E}_{\text {tot }}$ reads

$$
\begin{aligned}
\mathcal{E}_{\mathrm{tot}}= & \sum_{j=1,2}\left[\frac{\hbar^{2}}{2 m}\left|\nabla \psi_{j}\right|^{2}+V_{\mathrm{ext}}(\mathbf{r})\left|\psi_{j}\right|^{2}+\frac{1}{2} g_{j j}\left|\psi_{j}\right|^{4}\right] \\
& +g_{12}\left|\psi_{1}\right|^{2}\left|\psi_{2}\right|^{2}+\mathcal{E}_{\mathrm{BMF}}\left(\psi_{1}, \psi_{2}\right),
\end{aligned}
$$

where $V_{\text {ext }}(\mathbf{r})$ is an external confining potential and $g_{j k}=$ $4 \pi \hbar^{2} a_{j k} / m$, with $a_{j k}$ the intra- (with $a_{j k} \propto \delta_{j, k}$ ) and interspecies $(j \neq k)$ scattering lengths, respectively, and $n_{j}=$ $\left|\psi_{j}\right|^{2}$ is the density of the species $j$. The beyond-mean-field term $\mathcal{E}_{\mathrm{BMF}}$ arises from the zero-point energy of Bogoliubov collective excitations $[19,45]$, namely,

$$
\mathcal{E}_{\mathrm{BMF}}=\frac{8}{15 \pi^{2}}\left(\frac{m}{\hbar^{2}}\right)^{3 / 2}\left(g_{11} n_{1}\right)^{5 / 2} f\left(\frac{g_{12}^{2}}{g_{11} g_{22}}, \frac{g_{22} n_{2}}{g_{11} n_{1}}\right),
$$

with $f(x, y)=\sum_{ \pm}\left[1+y \pm \sqrt{(1-y)^{2}+4 x y}\right]^{5 / 2} / 4 \sqrt{2}$. In this section the calculation leading to the ground-state properties can be simplified by assuming the two components occupying the same spatial mode $[19,20]$. The bosonic fields can then be redefined as $\psi_{j}=\sqrt{n_{j}} \phi(\mathbf{r}, t)$. This assumption neglects the intercomponent dynamics and thus is inadequate to probe, for example, spin-dipole oscillations. In Sec. IV, within the variational framework, we present a modified Gaussian ansatz to include this feature in our study. The minimum of the meanfield energy density (3) fixes the ratio between the components of the population at which the spatial overlap is maximized, i.e., $N_{1} / N_{2}=\sqrt{a_{22} / a_{11}}[19,25]$, which we assume throughout this section. By defining $\Delta a=a_{12}+\sqrt{a_{11} a_{22}}$, Eq. (2) can then be expressed as

$$
\begin{aligned}
\mathcal{E}_{\mathrm{tot}}= & \frac{\hbar^{2} n_{\mathrm{tot}}}{2 m}|\nabla \phi|^{2}+n_{\mathrm{tot}} V_{\mathrm{ext}}(\mathbf{r})|\phi|^{2} \\
& +\frac{4 \pi \hbar^{2}}{m} \frac{\Delta a \sqrt{a_{22} / a_{11}}}{\left(1+\sqrt{a_{22} / a_{11}}\right)^{2}} n_{\mathrm{tot}}|\phi|^{4}+\frac{256 \sqrt{\pi} \hbar^{2}}{15 m} \\
& \times\left(\frac{n_{\mathrm{tot}} \sqrt{a_{11} a_{22}}}{1+\sqrt{a_{22} / a_{11}}}\right)^{5 / 2} f\left(\frac{a_{12}^{2}}{a_{11} a_{22}}, \sqrt{\frac{a_{22}}{a_{11}}}\right)|\phi|^{5},
\end{aligned}
$$

where $n_{\text {tot }}=n_{1}+n_{2}$. Inspired by the experiment described in [26], from now on we consider a quasi-one-dimensional optical waveguide. Then we take a harmonic confinement on a transverse plane, so $V_{\text {ext }}=\frac{1}{2} m \omega_{\perp}^{2}\left(x^{2}+y^{2}\right)$. The presence of a harmonic potential defines a characteristic length scale, namely, $a_{\perp}=\sqrt{\hbar / m \omega_{\perp}}$. In the following, all lengths are in units of $a_{\perp}$ and energies in units of $\hbar \omega_{\perp}$. Scattering length will be rescaled in units of the Bohr radius $a_{0}$ for convenience. The beyond-mean-field diagram and the static properties of the ground-state configurations have been studied in [26].

The properties of the system can be analytically explored by taking a Gaussian ansatz

$$
\phi(\mathbf{r})=\sqrt{\frac{L^{3}}{\pi^{3 / 2} \sigma_{r}^{2} \sigma_{z}}} \exp \left(-\sum_{r_{i}=x, y, z} \frac{r_{i}^{2}}{2 \sigma_{r_{i}}^{2}}\right)
$$

whose variational parameters are $\sigma_{r}$ and $\sigma_{z}$. The choice of normalization factor ensures that the original condition $\|\psi\|^{2}=N$ is preserved. By replacing Eq. (5) in Eq. (4) and taking the infinite-volume limit, the variational energy per particle is given by [26]

$$
\begin{aligned}
\frac{E}{N \hbar \omega_{\perp}}= & \frac{1}{4}\left(\frac{1}{\tilde{\sigma}_{x}^{2}}+\frac{1}{\tilde{\sigma}_{y}^{2}}+\frac{1}{\tilde{\sigma}_{z}^{2}}\right)+\frac{\tilde{\sigma}_{x}^{2}+\tilde{\sigma}_{y}^{2}}{4} \\
& +\frac{2 N \Delta \tilde{a}}{\sqrt{2 \pi} \tilde{\sigma}_{x} \tilde{\sigma}_{y} \tilde{\sigma}_{z}} \frac{\sqrt{\tilde{a}_{22} / \tilde{a}_{11}}}{\left(1+\sqrt{\tilde{a}_{22} / \tilde{a}_{11}}\right)^{2}}
\end{aligned}
$$




$$
\begin{aligned}
& +\frac{512 \sqrt{2}}{75 \sqrt{5} \pi^{7 / 4}} \frac{N^{3 / 2}}{\left(\tilde{\sigma}_{x} \tilde{\sigma}_{x} \tilde{\sigma}_{z}\right)^{3 / 2}}\left(\frac{\sqrt{\tilde{a}_{11} \tilde{a}_{22}}}{1+\sqrt{\tilde{a}_{22} / \tilde{a}_{11}}}\right)^{5 / 2} \\
& \times f\left(\frac{\tilde{a}_{12}^{2}}{\tilde{a}_{11} \tilde{a}_{22}}, \sqrt{\frac{\tilde{a}_{22}}{\tilde{a}_{11}}}\right) .
\end{aligned}
$$

The tilde signals that we are expressing a length in units of $a_{\perp}$. By means of Feshbach resonance [46], below a critical value of the magnetic field, the condition $\Delta a<0$ is achieved. Here, due to the simultaneous presence of quantum fluctuations and external confinement, two different self-bound states can be observed. First, for low particles number or, equivalently, small values of $|\Delta a|$, the ground state of the system corresponds to the so-called solitonic state, whose shape basically depends on the external trapping. Indeed, $\sigma_{x}=\sigma_{y} \sim a_{\perp}$, while the longitudinal one $\sigma_{z}$ is much greater. It is important to note the fact that beyond-mean-field corrections are not necessary for the stability of this state. Indeed, quantities such as the energy and density profile are in good agreement with the mean-field solutions for bright solitons in BECs [47].

The situation changes by increasing the particle number or lowering $\Delta a$. Here the system moves to an isotropic ground state $\left(\sigma_{x}=\sigma_{y}=\sigma_{z}<a_{\perp}\right)$, effectively independent of the confinement aspect ratios. The existence of this self-bound state is enabled by taking into account the contribution of Gaussian quantum fluctuations in the variational energy of Eq. (3). These results are summarized in Fig. 1, where we plot the width as a function of $N$ at fixed values of $\Delta a$ [Figs. 1(a)-1(c)] and in turn as a function of $\Delta a$ for chosen values of $N$ [Figs. 1(d)-1(f)] computed via the Gaussian ansatz (5).

At fixed $\Delta a$, the system approaches the droplet state by increasing the particle number. In order to reach a pure isotropic state, the effective mean-field attraction, i.e., $\Delta a$, has to be strong enough. For example, at $\Delta a=-5.1 a_{0}, \sigma_{z}$ remains two times larger than the radial width even at $N=10000$, while for $\Delta a=-10.6 a_{0}$ the system approaches a spherical configuration already at approximately 5000 particles. For weak attractive interactions (small negative $\Delta a$ ) the shift from one bound state to the other occurs via a smooth crossover (see, e.g., Fig. 1 for $\Delta a=-8.7 a_{0}$ ). At stronger interactions (large negative $\Delta a$ ) the system undergoes a bistability where competing minima are present in the energy functional and lead to a sharp structural change of the condensate as in Fig. 1 at $\Delta a=-10.6 a_{0}$ [26]. A similar picture emerges by considering a fixed particle number and tuning the effective mean-field attraction as in Figs. 1(d)-1(f).

\section{COLLECTIVE MODES IN THE VARIATIONAL APPROACH}

A deeper insight into the differences between solitonic and droplet states can be reached by examining collective excitations frequencies around the minimum of the variational energy of Eq. (6), given the Gaussian ansatz (5). The variational Gaussian approach ends up being particularly reliable in predicting collective excitations frequencies for a wide range of superfluid systems. As an example, in [34] a detailed analysis has been carried out by comparing the variational approach with numerical solutions of the Gross-Pitaevskii equation in purely 1D binary Bose mixtures. Concerning excitation frequencies, numerical and analytical predictions are shown to be in excellent agreement. Moreover, our theoretical framework was also applied to study droplet configurations and surface effects in confined fermionic superfluids as in [48], where variational results are well reproduced by density-functionaltheory simulations. By solving the eigenvalue problem of the Hessian matrix $\mathcal{H}(E)=\frac{\partial^{2} E}{\partial \sigma_{i} \partial \sigma_{j}}$ computed in the solitonic (or droplet) minimum, three different oscillation modes are allowed. In Fig. 2 we represent the three oscillation frequencies $\left(\omega_{I}, \omega_{I I}\right.$, and $\left.\omega_{I I I}\right)$ as predicted by the Gaussian ansatz.
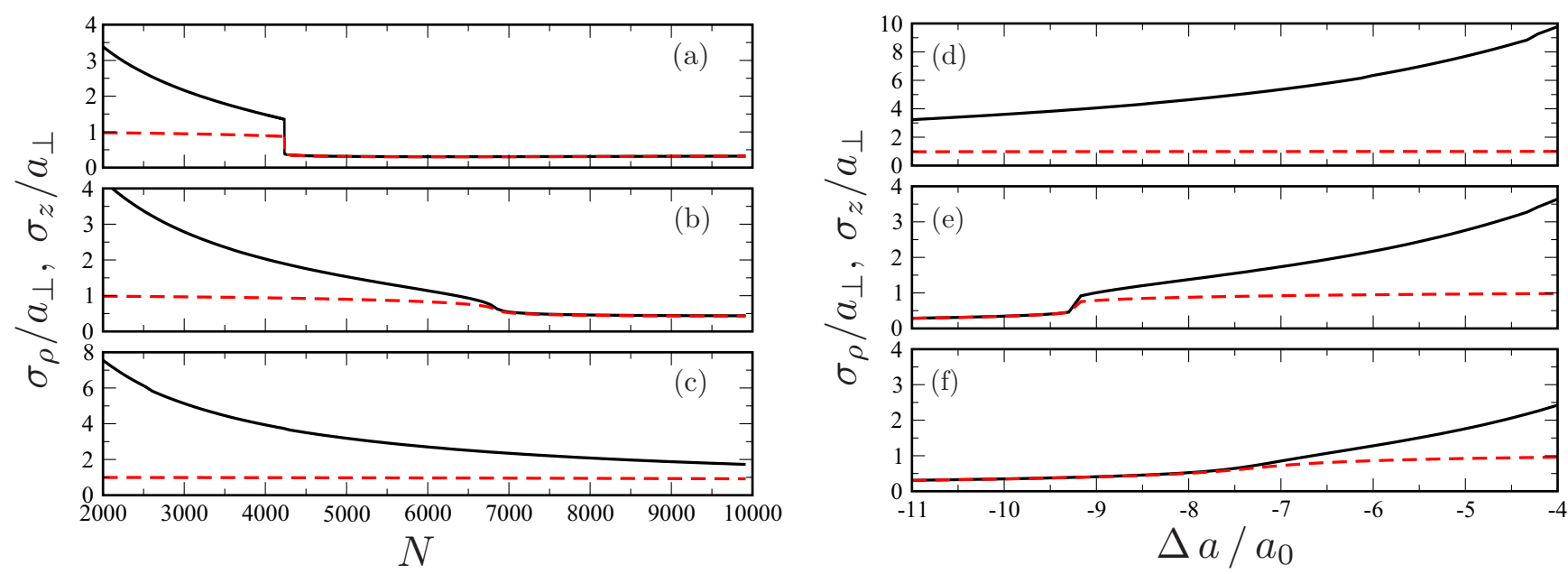

FIG. 1. Widths of the ground-state energy predicted by the variational Gaussian energy in Eq. (6). Widths $\sigma_{i}$ with $i=x, y, z$ are expressed in units of the oscillator length $a_{\perp}=\sqrt{\hbar / m \omega_{\perp}}$ for several values of $\Delta a\left(a_{0}\right)$ (units of Bohr's radius). Plotted are $\sigma_{z}$ (solid black line), the Gaussian width along the longitudinal direction, and $\sigma_{\rho}$ (red dashed line) along the transverse plane. Due to cylindrical symmetry of the confinement, $\sigma_{x}=\sigma_{y}$. The variational widths are plotted as a function of the particle number $N$ for (a) $\Delta a=-10.6 a_{0}$, (b) $\Delta a=-8.7 a_{0}$, and (c) $\Delta a=-5.1 a_{0}$. In (a) a sharp change of $\sigma_{r}$ and $\sigma_{z}$ is observed for $N \approx 4200$, signaling a bistability. Also shown are the variational widths as a function of $\Delta a$ for (d) $N=2000$, (e) $N=6000$, and (f) $N=10000$. 

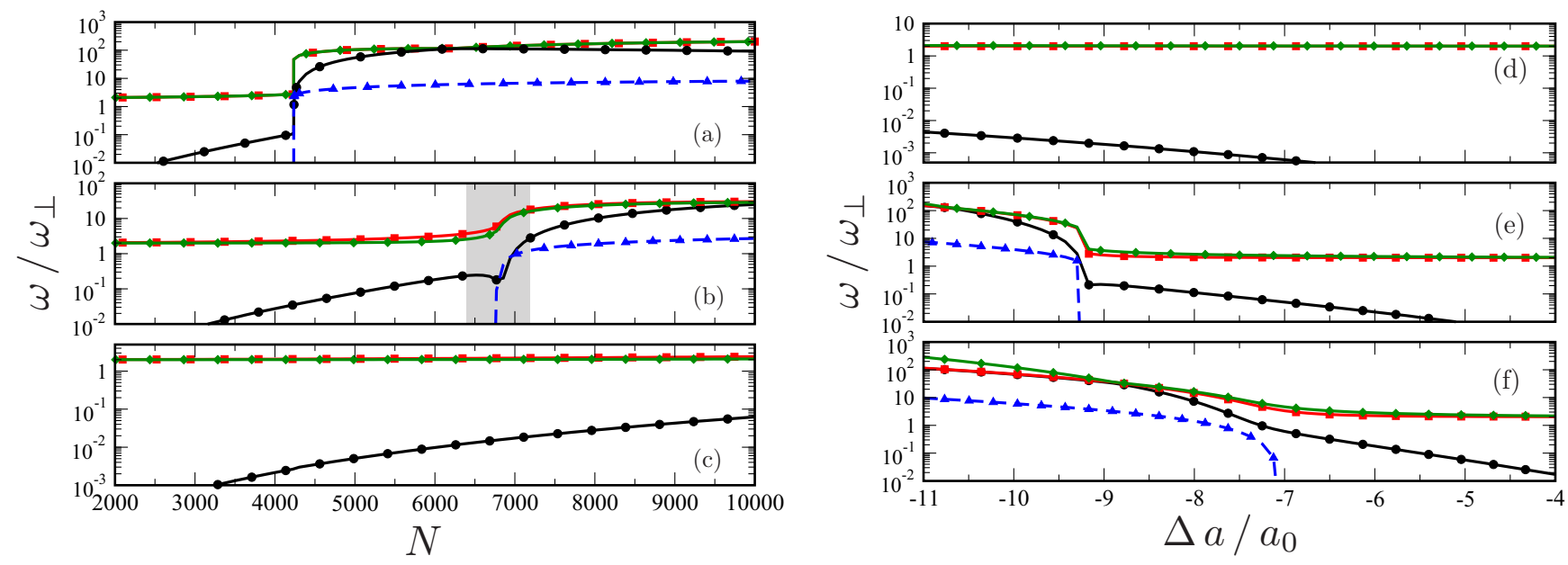

FIG. 2. Excitation frequencies $\omega_{I}, \omega_{I I}$, and $\omega_{I I I}$ and the particle-emission threshold $-\mu$, in units of $\hbar \omega_{\perp}$ as functions of $\Delta a$ in units of Bohr's radius $a_{0}$. The emission threshold (blue triangles) is shown only on the droplet side of the crossover. The excitation frequencies correspond to the eigenvalues of the Hessian matrix of Eq. (6). Frequencies are shown as a function of the particle number $N$ for the parameter (a) $\Delta a=-10.6 a_{0}$, (b) $\Delta a=-8.7 a_{0}$, and (c) $\Delta a=-5.1 a_{0}$. In (b) the gray region denotes the interval of particle numbers where the monopole mode is lower than the particle emission spectrum in the droplet phase. See Fig. 3 for a magnification of this area. Also shown are the frequencies as a function of $\Delta a$ for (d) $N=2000$, (e) $N=6000$, and (f) $N=10000$.

In the solitonic state one frequency, labeled $\omega_{I}$, can be easily excited, reflecting the absence of confinement along a direction. The other two modes are degenerate and converge to 2 (in units of $\hbar \omega_{\perp}$ ) as in a weakly interacting BEC [47]. The degeneracy of $\omega_{I I}$ and $\omega_{I I I}$ is an obvious consequence of the cylindrical symmetry of the radial confinement. This structure is clear in Fig. 2(d), where mode frequencies are depicted for the $N=2000$ case: $\omega_{I I}=\omega_{I I I} \simeq 2$, two order of magnitude higher than $\omega_{I}$. The same feature survives by increasing the particles number [Figs. 2(b), 2(c), 2(e), and 2(f)] on the solitonic side of the crossover for low values of $\Delta a$.

On the droplet side of the crossover, Fig. 2 also shows the particle emission threshold $-\mu$. The chemical potential $\mu$ is obtained by differentiating the total energy (4) with respect to $N$ within the Gaussian ansatz (5). In the solitonic state, $\mu>0$ for every value of $\Delta a$ and lies above $\omega_{I}$ and consequently this frequency can be excited and experimentally revealed. The eigenvector corresponding to $\omega_{I}$ displays the monopole character of this excitation, since $\mathbf{v}_{I}= \pm \alpha(1,1,1)^{T}$. Moving to the droplet side of the crossover, frequencies lie above the emission threshold, in agreement with the prediction of $[19,27]$. This implies that excitations of the system are damped by expelling particles from the droplet in a peculiar selfevaporation process. We also verified that, for $\Delta a=-5.1 a_{0}$, as in Fig. 2(c), upon increasing the particle number to larger values one enters the self-evaporation regime $\left(5.2 \times 10^{4}<\right.$ $N<1.4 \times 10^{5}$ ). Increasing even more the system size, selfevaporation ceases and the excitations are, at least in principle, observable. It is also interesting to observe that droplet length increases almost linearly with the particle number already for $N \geqslant 4 \times 10^{4}$.

\section{SPIN-DIPOLE OSCILLATIONS}

In this section we consider the relative dynamics between the two components of the mixture. We describe the oscillatory motion occurring when the two components are displaced from the center of the harmonic trap. To model their collective dynamics, we go beyond the assumption where the two components occupy the same spatial mode. We may introduce a weak confinement also along the $z$ axis with the corresponding aspect ratio $\lambda_{z}=\omega_{z} / \omega_{\perp}$.

Then we define a center-of-mass coordinate of each of the two components. The resulting Gaussian variational ansatz now reads

$$
\psi_{j}=\sqrt{\frac{N_{j}}{\pi^{3 / 2} \sigma_{r}^{2} \sigma_{z}}} e^{-\left(x^{2}+y^{2}\right) / 2 \sigma_{r}^{2}} \exp \left[-\frac{\left(z-z_{j}\right)^{2}}{2 \sigma_{z}^{2}}+i \alpha_{j} z\right],
$$

where the fields $\psi_{1}$ and $\psi_{2}$ obey the normalization condition $\int d^{3} \mathbf{r}\left|\psi_{j}\right|^{2}=N_{j}$. In Eq. (7) we assumed cylindrical symmetry $\sigma_{x}=\sigma_{y}=\sigma_{r}$. Here $\left\{\alpha_{i}\right\}_{i=1,2}$ describe the corresponding slopes of $\left\{z_{i}\right\}_{i=1,2}$. The set of variational parameters is then given by $\left\{z_{1}, z_{2}, \alpha_{1}, \alpha_{2}\right\}$.

Substituting (7) for the Euclidean action in Eq. (1), we derive the Lagrangian

$$
\begin{aligned}
L= & \sum_{j}\left\{-\hbar N_{j} z_{j} \dot{\alpha}_{j}-\frac{\hbar^{2} N_{j}}{2 m} \alpha_{j}^{2}-\frac{\hbar^{2} N_{j}}{2 m}\left(\frac{1}{\sigma_{r}^{2}}+\frac{1}{2 \sigma_{z}^{2}}\right)\right. \\
& \left.-\frac{N_{j}}{4} m \omega_{0}^{2}\left(2 \sigma_{r}^{2}+\lambda_{z}^{2} \sigma_{z}^{2}+2 \lambda_{z}^{2} z_{j}^{2}\right)-\frac{1}{2} \frac{g_{j j} N_{j}^{2}}{2 \sqrt{2} \pi^{3 / 2} \sigma_{r}^{2} \sigma_{z}}\right\} \\
& -\frac{g_{12} N_{1} N_{2} \exp \left[-\frac{\left(z_{j}-z_{k}\right)^{2}}{2 \sigma_{z}^{2}}\right]}{2 \sqrt{2} \pi^{3 / 2} \sigma_{r}^{2} \sigma_{z}}+\mathcal{E}_{\mathrm{BMF}},
\end{aligned}
$$

where the quantum fluctuations contribution in Eq. (3) is simplified by assuming $g_{12}^{2}=g_{11} g_{22}$, meaning that we are dealing with the system close to the mean-field instability threshold [49]. The beyond-mean-field term in the action then 
reads

$$
\mathcal{E}_{\mathrm{BMF}}=\left(\frac{m}{\hbar^{2}}\right)^{3 / 2} \int d^{3} \mathbf{r}\left(g_{11}\left|\psi_{1}\right|^{2}+g_{22}\left|\psi_{2}\right|^{2}\right)^{5 / 2} .
$$

In addition, to keep the calculation as analytical as possible, we consider a symmetric mixture, by taking $g_{11}=g_{22}$ with the corresponding equilibrium numbers $N_{1}=N_{2}$. Given the vector of variational parameters $\mathbf{q}$, the Euler-Lagrange equations are computed from Eq. (8) via $\frac{d}{d t} \frac{\partial L}{\partial \dot{q}_{i}}-\frac{\partial L}{\partial \mathbf{q}_{i}}=0$. Accordingly, the equation for the slope $\alpha_{i}=m \dot{z}_{i} / \hbar$ is elementary, so its dynamics is completely determined by that of $z_{i}$. A closed system of (linearized) differential equation can thus be derived straightforwardly

$$
\begin{aligned}
\ddot{z}_{1}+\omega_{0}^{2} \lambda_{z}^{2} z_{1}= & \sqrt{\frac{2}{\pi}}\left(\frac{a_{12} \hbar^{2} N_{1}}{m^{2} \sigma_{r}^{2} \sigma_{z}^{3}}\right)\left(z_{1}-z_{2}\right) \\
& -\frac{1024}{25 \pi^{1 / 4}}\left(\frac{a_{11}^{5 / 2} \hbar^{2} N_{1}^{3 / 2}}{m^{2} \sigma_{r}^{3} \sigma_{z}^{7 / 2}}\right)\left(z_{1}-z_{2}\right)\left(z_{1} \rightleftharpoons z_{2}\right) .
\end{aligned}
$$

The equation for $z_{2}$ has the same structure except for a global sign on the right-hand side of (10). The sum of these two equations leads to the equation for the longitudinal motion of the center of mass oscillating with the trap frequency $\omega_{0} \lambda_{z}$ (Kohn's theorem).

Concerning the relative coordinate $\tilde{z}=z_{1}-z_{2}$, we get

$$
\ddot{\tilde{z}}+\omega_{\text {rel }}^{2} \tilde{z}=0 .
$$

By rescaling the length in units of $a_{\perp}$ and the energies in units of $\hbar \omega_{\perp}$, the frequency $\omega_{\text {rel }}$ of the spin-dipole oscillations reads (in units of $\omega_{\perp}^{-1}$ )

$$
\begin{aligned}
\frac{\omega_{\mathrm{rel}}^{2}}{\omega_{\perp}^{2}}= & \lambda_{z}^{2}-\sqrt{\frac{8}{\pi}} \frac{N_{1}\left(a_{12} / a_{\perp}\right)}{\sigma_{r}^{2} \sigma_{z}^{3}} \\
& +\frac{2048}{25 \pi^{1 / 4}} \frac{\left(a_{11} / a_{\perp}\right)^{5 / 2} N_{1}^{3 / 2}}{\sigma_{r}^{2} \sigma_{z}^{7 / 2}},
\end{aligned}
$$

which, in absence of longitudinal confinement (i.e., $\lambda_{z}=$ 0 ), is always a positive defined quantity when the effective mean-field interaction is attractive $(\Delta a \lesssim 0)$. The results of the numerical analysis of the spin-dipole frequencies are summarized in Fig. 4 for a wide range of interactions and different particle numbers. We observe that only in the soliton regime do spin oscillations become observable $(N=1000)$. In the droplet phase self-evaporation is the dominant mechanism.

Concerning Eq. (12), we highlight the different sources of the two terms contributing to spin-dipole oscillations. The first one represents the mean-field attraction between the two components and thus depends only on $a_{12}$. Since we are considering the mutual dynamics between the components, it is reasonable that purely intracomponent terms do not contribute to $\omega_{\text {rel }}$. One has to consider however that Gaussian fluctuations are encoded in Eq. (9) in such a way that it is not possible to split it into two terms, each one referring to one component.

Equation (12) provides another peculiar feature of selfbound states in binary Bose mixtures: The occurrence of spin-dipole oscillations is not inhibited by turning off the longitudinal confinement, i.e., $\lambda_{z}=0$. Indeed, the interplay between

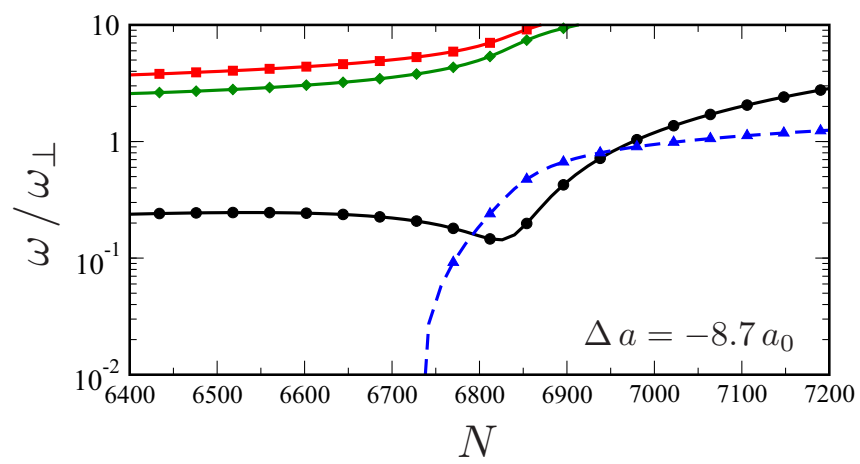

FIG. 3. Excitation spectrum at the crossover soliton droplet at $\Delta a=-8.7 a_{h o}$, shown as a magnification of the gray region of Fig. 2(b) with $N=6400-7200$. In the range $6780 \lesssim N \lesssim 6950$ monopole oscillations (black line with dots) are higher than the particle emission spectrum (dashed blue line with triangles).

the mean-field intercomponent attraction and the repulsion arising from quantum fluctuations establishes a restoring force. Then, differently from the mean-field scenario depicted in [44], the presence of a longitudinal confinement is not essential for spin-dipole oscillations to take place due to the attractive nature of the mean-field interaction between the two species.

\section{EXPERIMENTAL NUMBERS}

Experiments on self-bound states of binary Bose mixtures have been performed by considering a mixture of ${ }^{39} \mathrm{~K}$ atoms in two different internal states $|1\rangle=\left|F=1, m_{F}=-1\right\rangle$ and $|2\rangle=\left|F=1, m_{F}=0\right\rangle$. By tuning an external magnetic field, Feshbach resonances provide the possibility of exploring a wide range of intra- and interparticle scattering lengths. More precisely [46], by tuning the magnetic field between $B=$ 54 and $57.5 \mathrm{G}$, where $a_{11}=33.5 a_{0}$ and $a_{12}=-53.6 a_{0}$, the variation of $a_{22}$ provides a whole range of values for $\Delta a$ from $-15 a_{0}$ to $10 a_{0}$. Collective oscillations in binary mixtures can be probed in an experimental setup similar to the one developed in [26]. The existence of the soliton-droplet crossover can be revealed by engineering a cigar-shaped mixture and then removing the trapping along the $z$ axis and observing that the system still has a well-defined longitudinal length.

Our analytical results are compatible with the occurrence of self-evaporation for a large interval of experimental parameters. Only in a small window in the parameters space do we have that where monopole or quadrupole collective oscillations can in principle be observed as highlighted in Fig. 3. Moreover, we developed our analysis in a zero-temperature framework, leaving unanswered the question about what happens for small but finite temperatures.

In order to test our analytical results concerning the spindipole oscillations in Eq. (12) and Fig. 3, we require a tighter range of experimental values. In particular, as in [33], we consider the symmetric mean-field ground state given by the condition $a_{11} \simeq a_{22} \simeq 33.5 a_{0}$ at $B=54.5 \mathrm{G}$, where $a_{12}=$ $-54 a_{0}$. Reference [44] reported the experimental protocol to probe spin-dipole oscillations after a careful tuning of intra- and intercomponent scattering lengths. Indeed, each components in an overlapping initial state can be displaced 


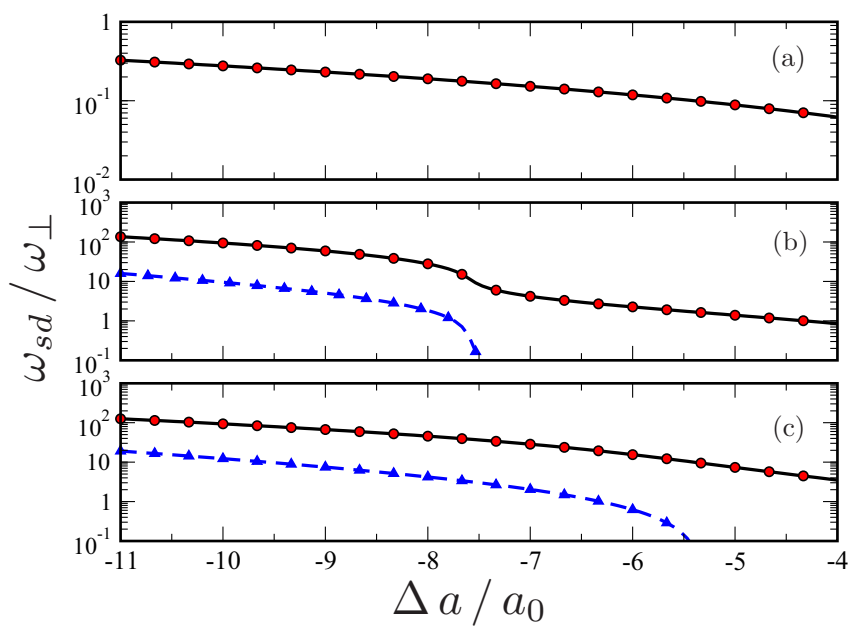

FIG. 4. Spin-dipole oscillations frequencies: spin-dipole oscillations frequency (red circles) and single-particle emission threshold $-\mu$ (blue triangles) for $N=1000,4000,10000$ particles. For $N=$ 1000 the chemical potential is positive in the range shown in the figure (soliton regime). In all cases the spin-dipole frequency is higher than $-\mu$ by more than one order of magnitude. The plots shown are done with equal intraspecies interaction $g_{11}=g_{22}$.

by applying a magnetic-field gradient $\delta B$ along the longitudinal axis, generated by two coils in an anti-Helmholtz configuration. Also, as stated in the preceding section, a longitudinal trapping is not mandatory to experimentally probe spin-dipole oscillations because of the peculiar structure of the Gaussian fluctuation contribution to the energy density and the attractive mean-field interaction. We emphasize that from an experimental perspective our study may provide insight into the observation of spin-dipole oscillations of solitons in a parameter regime which is complementary to that of [44]. Finally, we mention that the effect of three-body losses could be included in a full description of the excitation dynamics. This can done with the inclusion of the term $-i \hbar \frac{L_{3}}{2}|\psi|^{4}$ on the right-hand side of the Gross-Pitaevskii equation [50]. A dedicated study is left for future work.

\section{CONCLUSION}

In this work we analyzed the behavior of collective modes of two-component BECs with attractive interparticle interactions along the crossover from soliton to self-bound droplets in a quasi-one-dimensional waveguide. We observed a sharp difference in the collective modes in the two regimes. In the soliton regime modes vary smoothly upon the variation of particle number or interaction strength. On the droplet side collective modes are inhibited by the emission of particles. This mechanism turns out to be dominant for a wide range of particle numbers and interactions. In a small window of particle number range and for intermediate interactions we find that a monopole frequency is likely to be observed. We have studied the spin-dipole modes for the case of equal intraspecies interactions and equal equilibrium particle numbers in the presence of a weak longitudinal confinement. We have found that such modes might again be unobservable as their frequency is higher than the particle emission spectrum by at least one order of magnitude in the droplet phase. In conclusion, we have found that collective modes provide a complementary way to characterize two-component BECs in an optical waveguide. Moreover, they provide a strong signature to distinguish between solitonlike configurations and self-bound droplets. We expect that our work, inspired by experiments by Cabrera and co-workers, may provide a useful indication for further experiments with multicomponent BECs.

\section{ACKNOWLEDGMENTS}

T.M. acknowledges CNPq for support through the project "Bolsa de produtividade em pesquisa" via Grant No. 311079/2015-6 and the hospitality of the Physics Department of the University of Padova and the University of L'Aquila, where part of this work was done. L.S. is grateful for partial support from the BIRD project "Superfluid properties of Fermi gases in optical potentials" of the University of Padova.
[1] M. R. Matthews, D. S. Hall, D. S. Jin, J. R. Ensher, C. E. Wieman, E. A. Cornell, F. Dalfovo, C. Minniti, and S. Stringari, Phys. Rev. Lett. 81, 243 (1998).

[2] D. S. Hall, M. R. Matthews, J. R. Ensher, C. E. Weiman, and E. A. Cornell, Phys. Rev. Lett. 81, 1539 (1998).

[3] D. S. Hall, M. R. Matthews, C. E. Weiman, and E. A. Cornell, Phys. Rev. Lett. 81, 1543 (1998).

[4] I. M. Khalatnikov, Sov. Phys. JETP 5, 542 (1957).

[5] V. P. Mineev, Sov. Phys. JETP 40, 338 (1975).

[6] A. F. Andreev and E. P. Bashkin, Sov. Phys. JETP 42, 164 (1976).

[7] S. Beattie, S. Moulder, R. J. Fletcher, and Z. Hadzibabic, Phys. Rev. Lett. 110, 025301 (2013).

[8] M.-S. Chang, Q. Qin, W. Zhang, L. You, and M. S. Chapman, Nat. Phys. 1, 111 (2005).

[9] T. Zibold, E. Nicklas, C. Gross, and M. K. Oberthaler, Phys. Rev. Lett. 105, 204101 (2010).

[10] G. J. Conduit, A. G. Green, and B. D. Simons, Phys. Rev. Lett. 103, 207201 (2009).
[11] L. Salasnich and V. Penna, New J. Phys. 19, 043018 (2017).

[12] S. Liberati, M. Visser, and S. Weinfurtner, Class. Quantum Grav. 23, 3129 (2006).

[13] P. E. Larre and N. Pavloff, Europhys. Lett. 103, 60001 (2013).

[14] S. Butera, P. Öhberg, and I. Carusotto, Phys. Rev. A 96, 013611 (2017).

[15] Y. Li, L. P. Pitaevskii, and S. Stringari, Phys. Rev. Lett 108, 225301 (2012).

[16] G. I. Martone, Y. Li, L. P. Pitaevskii, and S. Stringari, Phys. Rev. A 86, 063621 (2012).

[17] Y. Li, G. I. Martone, L. P. Pitaevskii, and S. Stringari, Phys. Rev. Lett. 110, 235302 (2013).

[18] J.-R. Li, J. Lee, W. Huang, S. Burchesky, B. Shteynas, F. C. Top, A. O. Jamison, and W. Ketterle, Nature (London) 543, 91 (2017).

[19] D. S. Petrov, Phys. Rev. Lett. 115, 155302 (2015).

[20] D. S. Petrov and G. E. Astrakharchik, Phys. Rev. Lett. 117, 100401 (2016). 
[21] H. Kadau, M. Schmitt, M. Wenzel, C. Wink, T. Maier, I. FerrierBarbut, and T. Pfau, Nature (London) 530, 194 (2016).

[22] I. Ferrier-Barbut, H. Kadau, M. Schmitt, M. Wenzel, and T. Pfau, Phys. Rev. Lett. 116, 215301 (2016).

[23] L. Chomaz, S. Baier, D. Petter, M. J. Mark, F. Wächtler, L. Santos, and F. Ferlaino, Phys. Rev. X 6, 041039 (2016).

[24] M. Schmitt, M. Wenzel, F. Bottcher, I. Ferrier-Barbut, and T. Pfau, Nature (London) 539, 259 (2016).

[25] C. R. Cabrera, L. Tanzi, J. Sanz, B. Naylor, P. Thomas, P. Cheiney, and L. Tarruell, Science 359, 301 (2018).

[26] P. Cheiney, C. R. Cabrera, J. Sanz, B. Naylor, L. Tanzi, and L. Tarruell, Phys. Rev. Lett. 120, 135301 (2018).

[27] G. Semeghini, G. Ferioli, L. Masi, C. Mazzinghi, L. Wolswijk, F. Minardi, M. Modugno, G. Modugno, M. Inguscio, and M. Fattori, arXiv:1710.10890.

[28] F. Wächtler and L. Santos, Phys. Rev. A 93, 061603 (2016).

[29] D. Baillie, R. M. Wilson, R. N. Bisset, and P. B. Blakie, Phys. Rev. A 94, 021602(R) (2016).

[30] R. N. Bisset, R. M. Wilson, D. Baillie, and P. B. Blakie, Phys. Rev. A 94, 033619 (2016).

[31] A. Macia, J. Sanchez-Baena, J. Boronat, and F. Mazzanti, Phys. Rev. Lett. 117, 205301 (2016).

[32] F. Cinti, A. Cappellaro, L. Salasnich, and T. Macrì, Phys. Rev. Lett 119, 215302 (2017).

[33] A. Cappellaro, T. Macrì, G. F. Bertacco, and L. Salasnich, Sci. Rep. 7, 13358 (2017).

[34] G. E. Astrakharchik and B. A. Malomed, arXiv:1803.07165.

[35] J. Nespolo, G. E. Astrakharchik, and A. Recati, New J. Phys. 19, 125005 (2017).

[36] Emergent Nonlinear Phenomena in Bose-Einstein Condensates: Theory and Experiments, edited by P. G. Kevrekidis, D. J.
Frantzeskakis, and R. Carretero-González, Springer Series on Atomic, Optical, and Plasma Physics Vol. 45 (Springer, Berlin, 2007).

[37] F. Dalfovo, S. Giorgini, L. P. Pitaevskii, and S. Stringari, Rev. Mod. Phys. 71, 463 (1999).

[38] N. Henkel, R. Nath, and T. Pohl, Phys. Rev. Lett. 104, 195302 (2010).

[39] T. Macrì, F. Maucher, F. Cinti, and T. Pohl, Phys. Rev. A 87, 061602(R) (2013).

[40] F. Cinti, T. Macrì, W. Lechner, G. Pupillo, and T. Pohl, Nat. Commun. 5, 3235 (2014).

[41] T. Macrì, S. Saccani, and F. Cinti, J. Low Temp. Phys. 177, 59 (2014).

[42] F. Wächtler and L. Santos, Phys. Rev. A 94, 043618 (2016).

[43] D. Baillie, R. M. Wilson, and P. B. Blakie, Phys. Rev. Lett. 119, 255302 (2017).

[44] T. Bienaimé, E. Fava, G. Colzi, C. Mordini, S. Serafini, C. Qu, S. Stringari, G. Lamporesi, and G. Ferrari, Phys. Rev. A 94, 063652 (2016).

[45] D. M. Larsen, Ann. Phys. (NY) 24, 89 (1963).

[46] C. D’ Errico, M. Zaccanti, M. Fattori, G. Roati, M. Inguscio, G. Modugno, and A. Simoni, New J. Phys. 9, 223 (2007).

[47] L. Salasnich, A. Parola, and L. Reatto, Phys. Rev. A 66, 043603 (2002).

[48] F. Ancilotto, L. Salasnich, and F. Toigo, J. Low Temp. Phys. 171, 329 (2013)

[49] L. Pitaevskii and S. Stringari, Bose-Einstein Condensation (Oxford University Press, New York, 2003).

[50] M. Wenzel, F. Böttcher, T. Langen, I. Ferrier-Barbut, and T. Pfau, Phys. Rev. A 96, 053630 (2017). 